ARTICLE

\title{
Plasmodium vivax infection compromises reticulocyte stability
}

\author{
Martha A. Clark1, Usheer Kanjee ${ }^{1}$, Gabriel W. Rangel[1] ${ }^{2}$, Laura Chery ${ }^{3}$, Anjali Mascarenhas ${ }^{4}$, Edwin Gomes ${ }^{4}$, \\ Pradipsinh K. Rathod ${ }^{3}$, Carlo Brugnara (1) ${ }^{5}$, Marcelo U. Ferreira ${ }^{6} \&$ Manoj T. Duraisingh (iD) ${ }^{1 凶}$
}

The structural integrity of the host red blood cell (RBC) is crucial for propagation of Plasmodium spp. during the disease-causing blood stage of malaria infection. To assess the stability of Plasmodium vivax-infected reticulocytes, we developed a flow cytometry-based assay to measure osmotic stability within characteristically heterogeneous reticulocyte and $P$. vivax-infected samples. We find that erythroid osmotic stability decreases during erythropoiesis and reticulocyte maturation. Of enucleated RBCs, young reticulocytes which are preferentially infected by $P$. vivax, are the most osmotically stable. P. vivax infection however decreases reticulocyte stability to levels close to those of RBC disorders that cause hemolytic anemia, and to a significantly greater degree than $P$. falciparum destabilizes normocytes. Finally, we find that $P$. vivax new permeability pathways contribute to the decreased osmotic stability of infected-reticulocytes. These results reveal a vulnerability of $P$. vivax-infected reticulocytes that could be manipulated to allow in vitro culture and develop novel therapeutics.

\footnotetext{
${ }^{1}$ Department of Immunology and Infectious Diseases, Harvard T. H. Chan School of Public Health, Boston, MA, USA. ${ }^{2}$ Department of Biochemistry and Molecular Biology, Pennsylvania State University, University Park, PA, USA. ${ }^{3}$ Department of Chemistry, University of Washington, Seattle, WA, USA.

${ }^{4}$ Malaria Evolution in South Asia (MESA)-International Centers of Excellence in Malaria Research (ICEMR), Goa Medical College, Bambolim, Goa, India.

${ }^{5}$ Department of Laboratory Medicine, Boston Children's Hospital, Boston, MA, USA. ${ }^{6}$ Department of Parasitology, Institute of Biomedical Sciences,

University of São Paulo, São Paulo, SP, Brazil. ${ }^{凶}$ email: mduraisi@hsph.harvard.edu
} 
T he unique biconcave shape and structural dynamics of red blood cells (RBCs) permit traversal of the circulatory system and the delivery of oxygen to tissues. RBC structural integrity and deformability is determined by the molecular and cellular functions of the RBC cytoskeleton and membrane transporters ${ }^{1}$. Characterizing RBC stability under osmotic stress is a powerful method for probing the structural properties of the cytoskeleton and membrane transporter activity. An example of the relationship between RBC structural integrity and osmotic stability is seen with naturally occurring polymorphisms in molecules such as PIEZO1 and Ankyrin-1, that disrupt cell volume regulation and cytoskeleton, respectively, and are associated with the clinically relevant RBC phenotypes of hereditary xerocytosis $(\mathrm{HX})^{2}$ and hereditary spherocytosis $(\mathrm{HS})^{3}$. These mutations manifest in structurally compromised RBCs with abnormal shapes (stomatocytes and spherocytes), premature hemolysis in vivo ${ }^{4}$ and exhibit abnormal sensitivity to hypotonic challenge, which is decreased in HX and increased in HS.

During the Plasmodium spp. intra-erythrocytic developmental cycle (IDC), parasite remodeling alters the physiology of the host RBC. In the case of the most well studied of these parasites, $P$. falciparum, changes include disruption of the cytoskeleton by parasite proteins inserted into the RBC membrane ${ }^{5}$ and increased plasma membrane permeability 6,7 that culminate in the infected RBC taking on a spherical shape ${ }^{8}$. These changes compromise the structural integrity of the host RBC, and accordingly decrease the osmotic stability of $P$. falciparum-infected $\mathrm{RBCs}^{6,7,9}$. The consequence of the reduced stability of $P$. falciparum-infected RBCs is an increased propensity of infected cells to be destroyed in circulation via clearance by the spleen as well as intravascular hemolysis ${ }^{10}$.

Plasmodium vivax, the most globally widespread of the malaria parasites infecting humans ${ }^{11}$, exhibits a strict tropism for reticulocytes ${ }^{12-14}$, the youngest of circulating RBCs. This is in contrast to $P$. falciparum, which infects both reticulocytes and mature normocytes. All $P$. falciparum osmotic stability studies have been done in the older, more abundant normocyte fraction. Furthermore, recent observations that $P$. vivax increases reticulocyte deformability $13,15,16$, while $P$. falciparum decreases normocyte deformability $5,15,17$ suggest that the pathophysiology of $P$. vivax reticulocyte infection is fundamentally different than $P$. falciparum normocyte infection. The absence of an in vitro culture system and appropriate experimental methods have impeded the study of how P. vivax impacts the structural stability of the host reticulocyte. Moreover, though reticulocytes ${ }^{18-20}$ and erythroid progenitors ${ }^{21}$ are known to be more resistant to hypotonic lysis than normocytes, the dynamics of osmotic stability during erythropoiesis and reticulocyte maturation are unknown. Understanding the impact of $P$. vivax infection on the structural integrity of the host reticulocyte has the potential to provide important insight into why a long-term in vitro culture system for $P$. vivax has yet to be established, as well as the in vivo fitness of $P$. vivax-infected reticulocytes.

Osmotic stability is traditionally assessed by measuring release of hemoglobin from bulk RBC preparations in increasingly hypotonic solutions ${ }^{22}$. This methodology cannot resolve the osmotic stability of specific RBC subpopulations within characteristically heterogeneous reticulocyte and $P$. vivax samples. Furthermore, using free hemoglobin as the readout for RBC osmotic stability is problematic as hemoglobin levels are in flux in both parasite-infected RBCs and uninfected reticulocytes, because Plasmodium spp. digest hemoglobin as they mature ${ }^{23}$, and reticulocytes are actively synthesizing hemoglobin ${ }^{24}$. Flow cytometry, with its ability to examine discrete cell subsets within heterogeneous populations, has the potential to better define RBC osmotic stability. Finally, though previous studies have demonstrated the capacity of flow cytometry to detect hemolysis ${ }^{25-28}$, the current strategies have not been used to examine the lysis of discrete RBC populations and are furthermore not amendable to collecting sufficient data on rare cell populations.

In this work, we have developed a flow cytometric osmotic stability assay to assess the osmotic stability dynamics within inherently heterogeneous reticulocyte and malaria-infected RBC populations. We observe that osmotic stability steadily decreases during erythroid differentiation and reticulocyte maturation. Of enucleated RBCs, the youngest $\mathrm{CD} 71+$ reticulocytes which $P$. vivax preferentially invades using the $\mathrm{CD} 71 /$ transferrin receptor molecule as an invasion ligand ${ }^{13,14,29}$, are the most osmotically stable. Examination of clinical $P$. vivax samples showed $P$. vivax infection destabilized the host reticulocyte to levels close to those observed for RBCs from individuals with hemolytic anemia. Furthermore, $P$. vivax-infected reticulocytes are significantly less stable than $P$. falciparum-infected normocytes. Finally, we find that the decreased stability of $P$. vivax-infected reticulocytes corresponded with the appearance of $P$. vivax new permeability pathways (NPPs). The observation that reticulocyte osmotic stability is reduced by $P$. vivax infection suggests that $P$. vivaxinfected reticulocytes may be prone to pre-mature hemolysis in vivo. These results reveal a key vulnerability of the parasite that if corrected may facilitate the adaptation of $P$. vivax to in vitro culture, and conversely if targeted could yield novel therapeutics to treat blood stage $P$. vivax infection.

\section{Results}

Flow cytometry analysis of RBC osmotic stability. While flow cytometry has been employed to study osmotic stability, previous studies have not harnessed the capacity of flow cytometry to analyze multiple cell populations within a single specimen ${ }^{25-28}$. Therefore, we developed a flow cytometric osmotic stability assay that yields the same hemolysis curves as the traditional hemoglobin absorbance assay and that by quantifying the individual cells that survive hemolysis challenge is able to assess osmotic stability dynamics within heterogeneous RBC populations (Fig. 1a). The analysis of hemolysis by flow cytometry revealed typical RBC forward scatter (FSC) and side scatter (SSC) profiles for non-lytic isotonic conditions, and the subsequent appearance of a low-FSC/low-SSC population in hypotonic, lytic conditions (Fig. $1 \mathrm{~b}$ and Supplementary Fig. 1).

To confirm that the low-FSC/low-SSC population were RBC ghosts, fluorescent phalloidin, which is excluded from intact cells but binds actin in the cytoskeleton of permeabilized cells was included in the osmotic stability assay. We found that phalloidin stained the FSC-low population that appears in lytic conditions, indicating that the flow lysis assay is sensitive to RBC ghosts (Fig. 1c and d). Considering only intact RBCs, the lysis curves produced by the flow lysis assay were indistinguishable from those of the hemoglobin absorbance lysis assay (Fig. 1e). No difference in the lysis ${ }_{50}$ values (mOsm at which $50 \%$ of RBCs lyse) obtained for normal RBCs using the two lysis assays confirmed the accuracy of the flow lysis assay. To test the limits of the flow lysis assay, we measured the osmotic stability of HX RBCs that are resistant to hypotonic lysis. With HX RBCs as well, we observed no difference in lysis 50 values generated by the two assays (Fig. 1f).

Finally, as $P$. vivax osmotic stability studies included cryopreserved samples and were performed on RBCs maintained in vitro, we examined the effect of (i) cold storage and (ii) P. vivax in vitro culture conditions on RBC osmotic stability. As expected $^{30,31}$, we observed variation in the osmotic stability of normal RBCs taken from 12 different donors with lysis ${ }_{50}$ values 
a.

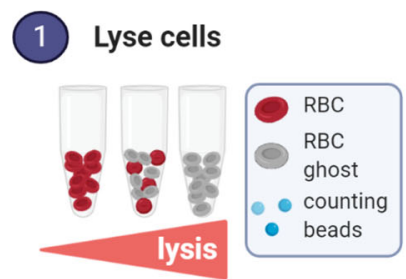

Quench lysis \& add counting beads

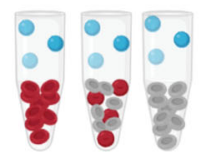

(3) Flow cytometry analysis of lysis

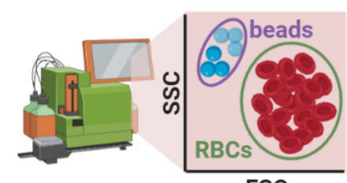

FSC b.

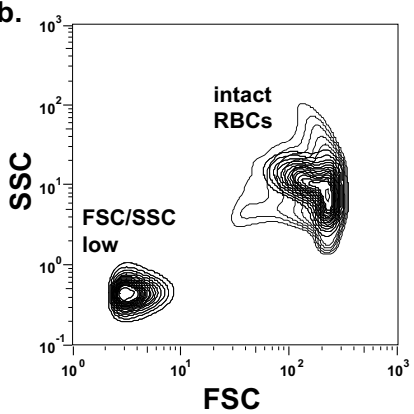

e.

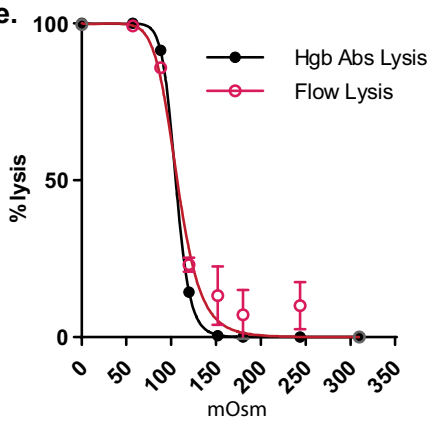

c.

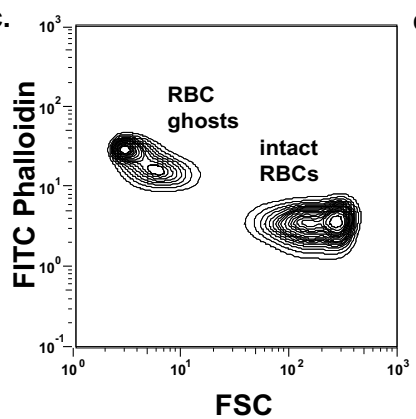

f.

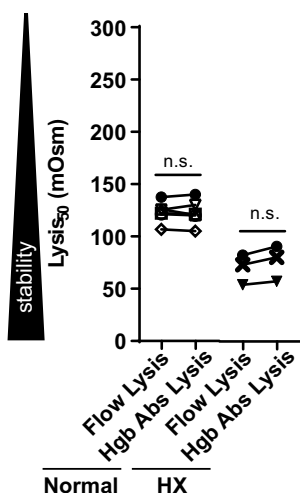

d.

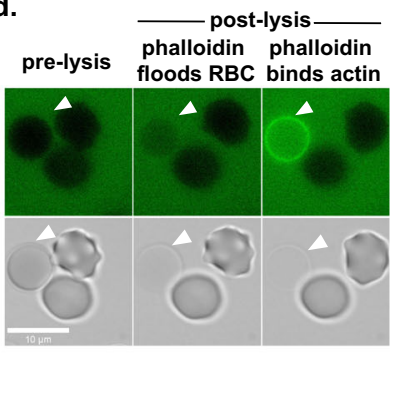

g.

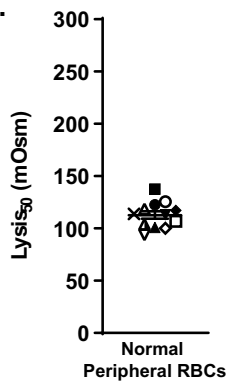

(4) Quantification of lysis

Lysis =1- $\frac{\left(\begin{array}{c}\text { RBCs recovered } \\ \text { from lytic } \\ \text { condition } \\ \text { counting } \\ \text { beads }\end{array}\right)}{\left(\begin{array}{c}\text { RBCs recovered } \\ \text { from non-lytic } \\ \text { control condition } \\ \text { counting } \\ \text { beads }\end{array}\right)}$ h.

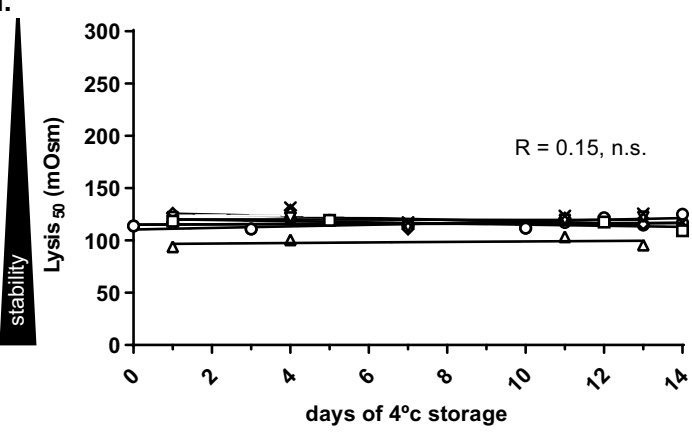

i.

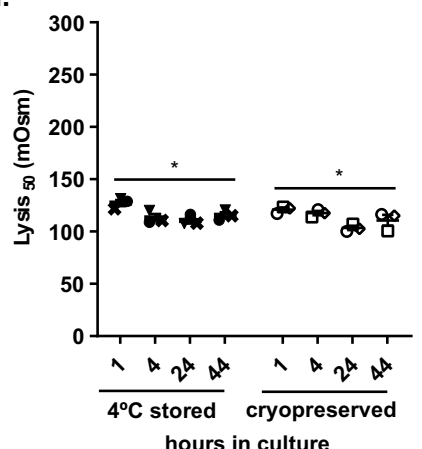

Fig. 1 Development of a flow cytometry osmotic stability assay. a Flow cytometry method for measuring RBC osmotic stability. Created with BioRender. com. $\mathbf{b}$ and $\mathbf{c}$ Representative flow cytometry forward scatter (FSC) side scatter by (SSC) plot (b) and FSC by FITC-phalloidin plot of a lysed (120 mOsm) RBC sample (c). Data representative of five independent experiments. d Representative immunofluorescent images of FITC-phalloidin binding RBC ghosts. Scale bar, $10 \mu \mathrm{m}$. Arrows indicate a RBC undergoing lysis. Data representative of two independent experiments. e Representative hemoglobin (Hgb) absorbance (black line) and flow cytometry (red line) lysis curves for normal RBCs. Error bars represent SD of $n=3$ technical replicates. Data fit with leastsquares regression fit curves of normalized data. $\mathbf{f} \operatorname{Normal}(n=6)$ and hereditary xerocytosis $(H X)(n=3)$ RBC lysis 50 values measured by flow cytometry and hemoglobin absorbance lysis assays. Unique symbols indicate biological replicates and lines match the lysis 50 values obtained from flow cytometry and hemoglobin absorbance assays. n.s. indicates no significant difference in lysis ${ }_{50}$ (normal RBC $p=0.8, \mathrm{HX}$ RBC $p=0.1$ ) using paired two-sided Student's $t$ test. $\mathbf{g}$ Normal RBC $(n=12)$ lysis 50 values measured by flow cytometry. Unique symbols indicate biological replicates. Horizontal lines and error bars represent mean \pm SEM. $\mathbf{h}$ Normal RBC $(n=6)$ lysis 50 values over the course of 14 days of $4^{\circ} \mathrm{C}$ storage. Unique symbols indicate biological replicates. Data fit with linear regression lines. Mean linear regression for all data depicted by solid line. Pearson correlation of lysis 50 and days of $4{ }^{\circ} \mathrm{C}$ storage, $r=0.15$. $\mathbf{i} 4{ }^{\circ} \mathrm{C}$ degree stored $(n=3)$ and cryopreserved $(n=3) \mathrm{RBCs}_{\text {lysis }}$ 50 values following transfer to $P$. vivax in vitro culture conditions. Unique symbols indicate biological replicates. Horizontal lines and error bars represent mean $\pm \mathrm{SEM}$. Asterisks denote significant differences in lysis 50 during culture $\left(4^{\circ} \mathrm{C}\right.$ degree stored RBCs $p=0.02$, Cryopreserved RBCs $p=0.04$ ) using RM one-way ANOVA analysis.

ranging from 93.9 to $137.9 \mathrm{mOsm}$ (variation of $11.2 \%$ ) (Fig. 1g). Subsequent storage of RBCs from six different donors at $4{ }^{\circ} \mathrm{C}$ for 2 weeks resulted in no appreciable change (mean slope $-0.063 \pm$ 0.24 SEM, Pearson $r=0.15$ ) in osmotic stability (Fig. 1h). However, when cryopreserved $\mathrm{RBCs}$ and $\mathrm{RBCs}$ stored at $4{ }^{\circ} \mathrm{C}$ were transferred to $P$. vivax in vitro culture conditions, osmotic stability increased by $13 \pm 2.6 \%$ and $14.6 \pm 0.8 \%$ after $24 \mathrm{~h}$ of incubation (Fig. 1I).

Osmotic stability decreases during RBC differentiation. Having established the capacity of flow cytometry to measure RBC osmotic stability, we next examined the osmotic stability dynamics within the RBC fractions that harbor $P$. vivax infection. To this end, we took advantage of the capacity of flow cytometry to track discrete cell populations, and separately assess the osmotic stability of nucleated RBC precursors, reticulocytes, and normocytes in bone marrow aspirates (Fig. 2a and Supplementary 2A). We found that fluorescent labeling of reticulocytes and nucleated $\mathrm{RBC}$ precursors present within bone marrow aspirates allowed us to quantitate the osmotic stability dynamics of each of these RBC subpopulations simultaneously (Fig. 2b). For nucleated RBC precursors we 


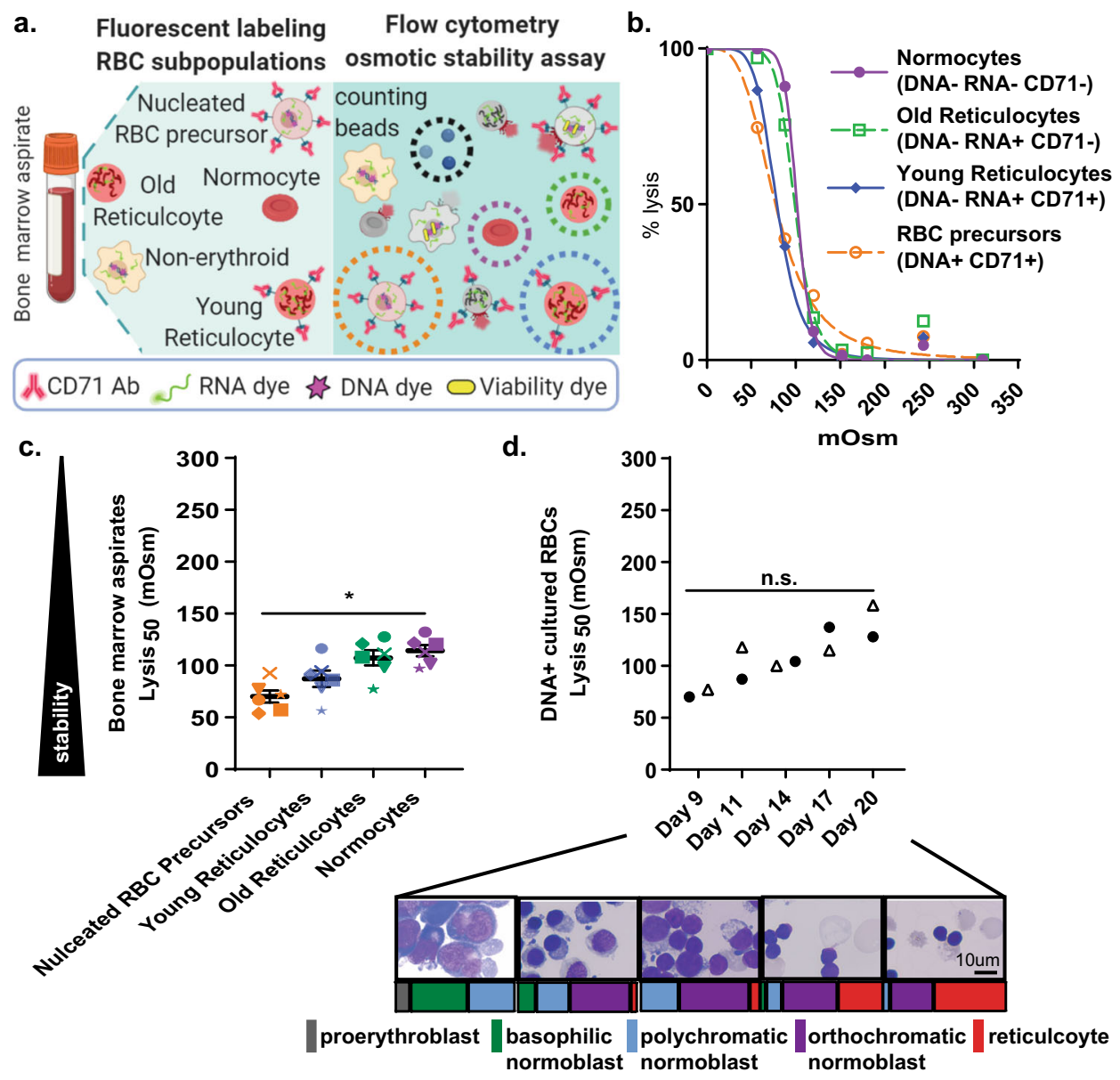

Fig. 2 Osmotic stability dynamics during erythroid differentiation and reticulocyte maturation. a Flow cytometry method for measuring osmotic stability of RBC subpopulations. Created with BioRender.com. b Representative lysis curves for normocytes (DNA- RNA- CD71-, purple line), old reticulocytes (DNA- RNA+ CD71, green dashed line), young reticulocytes (DNA- RNA+ CD71+, blue solid line), and RBC precursors (DNA+ CD71+, orange dashed line) from a bone marrow aspirate measured by flow cytometry. Data fit with least squares regression fit curves of normalized data. c Normocyte (purple data points), old reticulocytes (green data points), young reticulocytes (blue data points), and RBC precursors (orange data points) from bone marrow aspirates lysis 50 values $(n=6)$. Unique symbol indicate biological replicates. Horizontal lines and error bars represent mean \pm SEM. Asterisks denote significant differences in lysis 50 values $(p=0.0001)$ using RM one-way ANOVA analysis. d RBC progenitor lysis 50 values during course of CD34+ differentiation in vitro $(n=2)$. Unique symbols indicate RBC progenitor lysis $5_{0}$ values at days $9,11,14,17$, and 20 from two independent differentiations. n.s. indicates no significant difference in lysis ${ }_{50}$ during course of differentiation $(p=0.2)$ using Friedman test. Inset are representative photos of RBC progenitors during in vitro differentiation. Scale bar, $10 \mu \mathrm{m}$. Colored bars below images represent proportion of RBC progenitor developmental stages (gray - proeythroblast, green-basophilic normoblast, blue-polychromatic normoblast, purple-orthochromatic normoblast, red-reticulocyte) present at day$9,-11,-14,-17$, and -20 of differentiation.

additionally found that lysed cells were also identifiable with a live/dead stain (Supplementary 2B). Importantly, as both osmotic stability studies with bone marrow aspirates and clinical $P$. vivax samples relied on Percoll enrichment to raise reticulocytemia and $P$. vivax parasitemia to reliably measurable levels, we found that Percoll had no effect on the osmotic stability of reticulocytes and nucleated precursors from bone marrow aspirates (Supplementary 2C).

The lysis 50 values obtained for RBC precursors and reticulocytes from bone marrow aspirates revealed that nucleated $\mathrm{RBC}$ precursors $(\mathrm{DNA}+\mathrm{CD} 71+$ ) were the most osmotically stable (Lysis $_{50} 70.1 \pm 5.8$ ) followed by the youngest CD71+ RNA+ DNA- reticulocytes $\left(\right.$ Lysis $_{50} 87.3 \pm 8.0$ ), older CD71- RNA+ DNA - reticulocytes (Lysis 50707.4 7.3) and CD71- RNADNA - normocytes (Lysis ${ }_{50} 114.5 \pm 5.4$ ) (Fig. $2 b$ and c). To establish osmotic stability dynamics during erythropoiesis, we assessed the osmotic stability of erythroid progenitors differentiated in vitro from CD34+ stem cells ${ }^{19,32}$. This study revealed a strong trend of decreasing osmotic stability as cells progressed in vitro from majority basophilic and polychromatic normoblasts at day 9 (Lysis $\left._{50} 73.8 \pm 3.6\right)$ to a majority polychromatic and orthochromatic normoblast population at day $11\left(\right.$ Lysis $_{50} 102.7 \pm$ 15.6), and then further decreased as cells progressed through final orthochromatic normoblast maturation occurring between day 14 (Lysis $_{50} 102.2 \pm 2.0$ ) day 17 (Lysis $_{50} 126.2 \pm 11.2$ ), and day 20 (Lysis 50 143.4 \pm 15.1 ) (Fig. 2d). Parallel examination of the osmotic stability of CD71+ reticulocytes differentiated in vitro showed no significant difference in osmotic stability as a function of day reticulocytes were sampled (Supplementary Fig. 2D). Furthermore, consistent with a previous study ${ }^{19}, \mathrm{CD} 71+$ reticulocytes generated in vitro (Lysis ${ }_{50} 110.1 \pm 3.7$ ) were less stable than $\mathrm{CD} 71+$ reticulocytes from bone marrow samples (Lysis $_{50}$ 87.3 \pm 8.0 ) (Supplementary Fig. 2E). Together these results demonstrate the capacity of flow cytometry to assess the osmotic stability of discrete RBC subsets within heterogeneous populations and clearly shows that erythroid development and reticulocyte maturation are associated with significant changes in osmotic stability. 
a.

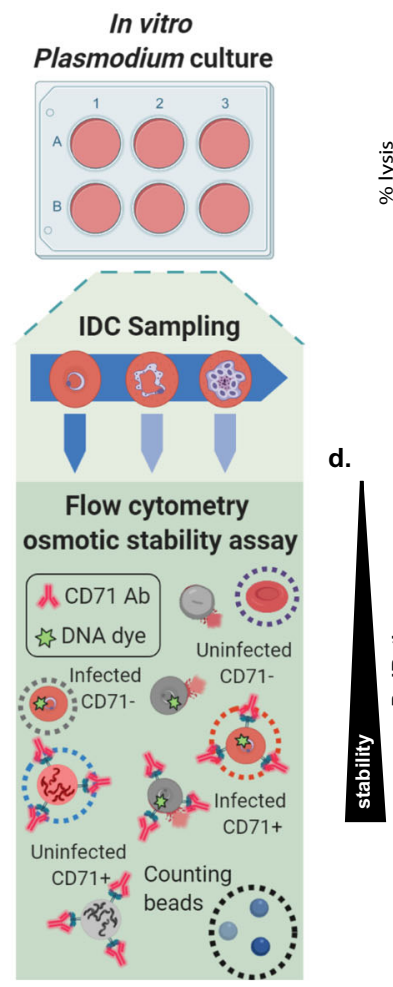

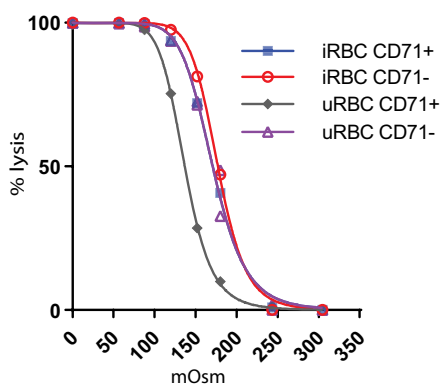

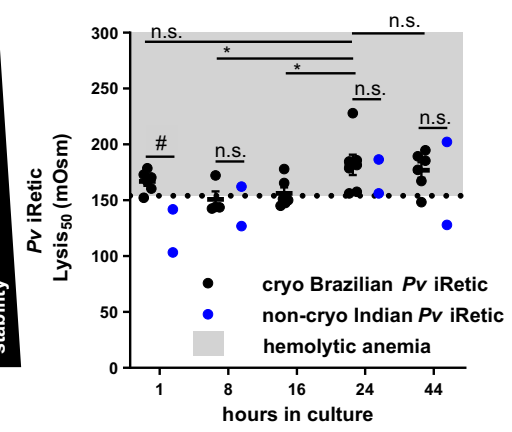

c.

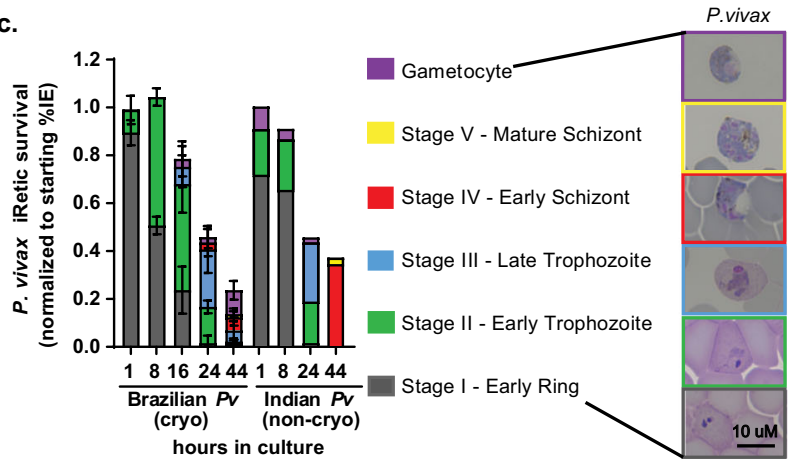

e.

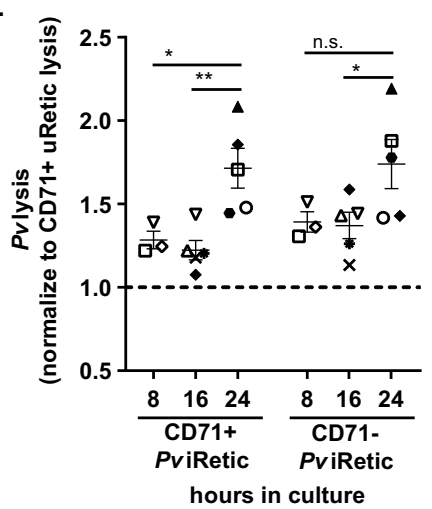

Fig. 3 Osmotic stability of $\boldsymbol{P}$. vivax-infected reticulocytes. a Flow cytometry strategy for measuring osmotic stability Plasmodium-infected RBCs. Created with BioRender.com. b Representative lysis curves for P. vivax-infected CD71+ (DNA+CD71+, blue line) and CD71- reticulocytes (DNA+ CD71-, red line) and uninfected CD71+ reticulocytes (DNA- CD71+, gray line) and normocytes (DNA- CD71-, purple line) from a cryopreserved Brazilian clinical P. vivax sample after 1-h in vitro culture. Data fit with least-squares regression fit curves of normalized data. $\mathbf{c}$ Developmental stage of in vitro cultured ex vivo Indian $(n=2)$ and cryopreserved Brazilian $(n=5)$ P. vivax clinical isolates. Horizontal lines and error bars represent mean \pm SEM. Inset are representative images of P. vivax IDC development stages. Scale bar, $10 \mu \mathrm{m}$. Gray (stage I), green (stage II), blue (stage III), red (stage IV), yellow (stage V), purple (gametocyte) bars indicate IDC development stage. d $P$. vivax-infected reticulocytes from cryopreserved Brazilian (black symbols) and non-cryopreserved Indian (blue symbols) clinical P. vivax samples lysis 50 values after 1-, 8-, 16-, 24-, and 44-h of in vitro culture. Unique symbols indicate biological replicates. Horizontal lines and error bars represent mean \pm SEM. Dashed line $(154 \mathrm{mOsm})$ and gray shading indicate lysis 50 threshold for hemolytic anemias. Hashtag and n.s., significant and no significant difference in cryopreserved Brazilian and non-cryopreserved Indian $P$. vivax lysis 50 values, respectively (1-h $p=0.05,8$-h $p=0.6,24-\mathrm{h} p=0.8$, 44$\mathrm{h} p=1.0$ ) using unpaired two-tailed Mann-Whitney test. Astericks and n.s., significant and no significant difference between Brazilian $P$. vivax-infected reticulocytes lysis 50 at 24- and 1-, 8-, 16-, 24- and 44-h of culture, respectively (1-h $p=0.3,8-\mathrm{h} p=0.02,16-\mathrm{h} p=0.04$, and 44-h $p=0.9)$ using unpaired two-tailed Dunnett's multiple comparisons test. e Cryopreserved Brazilian $P$. vivax-infected CD71+ and CD71- lysis 50 values normalized to lysis 50 values of uninfected CD71+ reticulocytes at 8- $(n=3), 16-(n=5)$, and 24-h $(n=5)$ of culture. Unique symbols indicate biological replicates. Horizontal lines and error bars represent mean \pm SEM. Astericks and n.s., significant and no significant difference between $24-h$ and 8 - and 16 - $h$ of culture lysis ${ }_{50}$ values, respectively (CD71+: 8-h $p=0.04,16-\mathrm{h} p=0.006$ and CD71-: 8-h $p=0.1,16-\mathrm{h} p=0.05$ ) using unpaired two-tailed Student's t-test.

P. vivax infection reduces reticulocyte osmotic stability. Having established that the youngest $\mathrm{CD} 71+$ reticulocytes that are preferred by $P$. vivax for invasion ${ }^{13,14}$ are the most osmotically stable of all enucleated RBCs, we next assessed the impact of $P$. vivax infection on reticulocyte osmotic stability. In the absence of continuous $P$. vivax in vitro culture, cryopreserved clinical $P$. vivax samples are an invaluable resource for investigating $P$. vivax biology ${ }^{33-36}$. Cognizant of the decreased stability of cryopreserved RBCs, however (Fig. 1i), we first directly compared the in vitro survival and osmotic stability of cryopreserved and noncryopreserved $P$. vivax clinical samples as parasites progressed through the IDC (Fig. $3 \mathrm{a}$ and $\mathrm{b}$ and Supplementary Fig. 3A). Consistent with previous studies ${ }^{33-36}$, we observed a $78 \pm 3.3 \%$ and $63 \pm 30.8 \%$ loss of $P$. vivax-infected reticulocytes prior to completion of the IDC in vitro (44-h cultures) for Brazilian cryopreserved and Indian non-cryopreserved clinical samples, respectively (Fig. 3c).

Parallel osmotic stability measurements of the uninfected RBCs in $P$. vivax clinical samples revealed that, as observed previously (Fig. 1I), the stability of cryopreserved uninfected RBC populations increased upon transfer to in vitro culture while non-cryopreserved uninfected RBCs remained steady during the course of culture (Supplementary Fig. 3B and C). For $P$. vivax-infected reticulocytes, we observed that cryopreserved stage I ring-infected reticulocytes were less stable than non-cryopreserved stage I rings. For subsequent time points we observed (i) no difference in the osmotic stability of cryopreserved and non-cryopreserved $P$. vivax-infected reticulocytes and (ii) a decrease in the stability of $P$. vivax-infected reticulocytes as they progressed through the IDC (Fig. 3d). Moreover, when we consider the clinical laboratory cutoff for normal RBCs lysis $_{50}$ $154 \mathrm{mOsm}$ or $0.45 \% \mathrm{NaCl})^{31,37,38}$, the osmotic stability of $88 \%$ of P. vivax-infected reticulocytes (cryopreserved and non-cryopreserved) in 24- and 44-h cultures fell into the range of osmotic instability associated with RBC disorders that cause hemolytic anemia such as HS.

We next examined the degree of instability $P$. vivax induced in the host reticulocyte by comparing the osmotic stability of 
a.

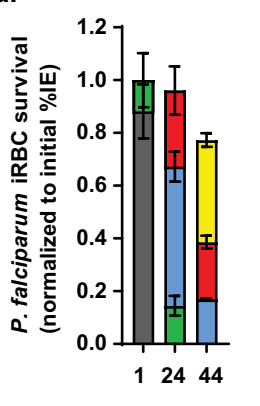

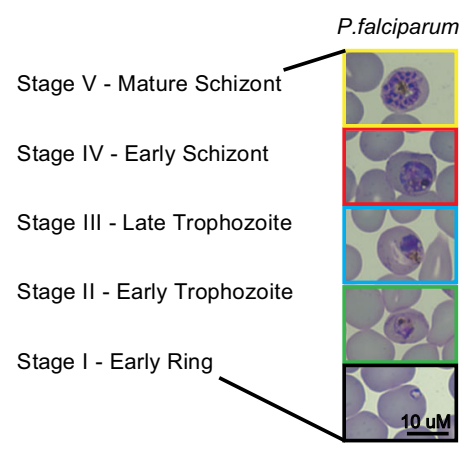

b.

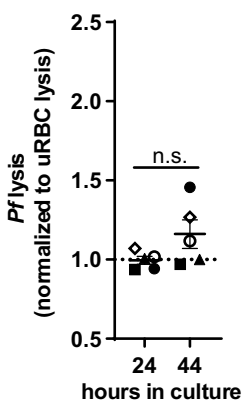

c.

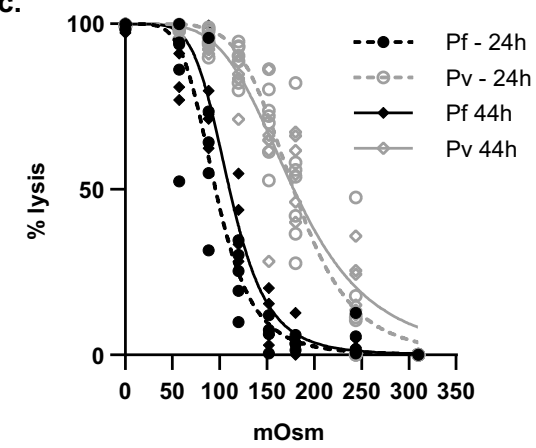

Fig. 4 Osmotic stability of P. falciparum-infected normocytes. a Developmental stage of in vitro-cultured, cryopreserved $P$. falciparum (clone 3D7 P2G12) $(n=5)$. Bars represent the mean \pm SEM. Inset are representative images of $P$. falciparum IDC development stages. Scale bar, $10 \mu \mathrm{m}$. Gray $($ stage I), green (stage II), blue (stage III), red (stage IV), yellow (stage V) bars indicate IDC development stage. b $P$. falciparum-infected RBC lysis 50 values normalized to lysis 50 values of corresponding uninfected RBCs at 24 - and $44-\mathrm{h}$ of in vitro culture of cryopreserved $P$. falciparum (clone 3D7 P2G12) ( $n=5$ ). Unique symbols indicate biological replicates. Horizontal lines and error bars represent mean \pm SEM. n.s., no significant difference between $P$. falciparum-infected RBC lysis 50 values at 24 and 44-h of culture $(p=0.1)$ using paired two-tailed Student's t-test. c Lysis curves of 24-h (gray dotted line) $(n=7)$ and $44-h$ (gray solid line) $(n=6)$ cryopreserved Brazilian P. vivax-infected reticulocyte cultures and 24-h (black dotted line) $(n=5)$ and $44-\mathrm{h}($ black solid line) $(n=$ 5) cryopreserved P. falciparum-infected normocytes (clone 3D7 P2G12) cultures. Unique symbols indicate biological replicates. Data fit with least-squares regression fit curves of normalized data.

CD71 - and CD71 + P. vivax-infected reticulocytes to that of uninfected $\mathrm{CD} 71+$ reticulocytes. To take advantage of the availability of cryopreserved $P$. vivax clinical samples while also minimizing the influence of cryopreservation, we excluded the 1 -h post thaw time point from subsequent analysis. Due to reticulocyte maturation ${ }^{39}$, this analysis was limited to the first 24$\mathrm{h}$ of in vitro culture, as the frequency of CD71+P. vivax-infected and uninfected reticulocytes, respectively, decreased by $56 \pm 8.0 \%$ and $64 \pm 3.5 \%$ between 1 and 24 -h of culture, and then fell below the limit of detection ( $0.05 \%$ CD71+ reticulocytes) between 24 and $44 \mathrm{~h}$. Of note, the persistence of $P$. vivax-infected CD71+ reticulocytes in our ex vivo cultures through $24 \mathrm{~h}$ (Supplementary Fig. 3D and $3 \mathrm{E}$ ) is longer than previously observed for CD71+ cord blood reticulocytes invaded in vitro by $P$. vivax ${ }^{13}$. The reason for this discrepancy is not immediately evident and therefore subject for future investigation. We found infected reticulocytes were less stable than uninfected $\mathrm{CD} 71+$ reticulocytes at all-time points assessed (8-, 16-, and 24-hour cultures), and the progression of $P$. vivax through the IDC further decreased reticulocyte osmotic stability with the appearance of stage III late trophozoite forms in 24-h cultures corresponding to $P$. vivax-infected CD71+ and CD71- reticulocytes being 71.4 $\pm 12.0 \%$ and $74.0 \pm 14.6 \%$ less stable than uninfected CD71+ reticulocytes (Fig. 3d). Finally, no difference in the stability of CD71+ and CD71 - infected-reticulocytes indicated that $P$. vivax infection and not reticulocyte age is the primary determinate of the stability of $P$. vivax-infected reticulocytes (Supplementary Fig. 3F).

$P$. vivax-infected reticulocyte are less stable than $P$. falciparuminfected normocytes. Considering previous observations that $P$. vivax and $P$. falciparum-infected RBCs exhibit different rheological properties $5,13,15-17$, we next compared $P$. vivax destabilization of reticulocytes with $P$. falciparum destabilization of normocytes. To account for the influence of cryopreservation on our $P$. vivax osmotic stability studies, we assessed the in vitro survival and osmotic stability of cryopreserved $P$. falciparum clone $3 D 7 \mathrm{P} 2 \mathrm{G} 12^{40}$. We observed similar progression of $P$. vivax and $P$. falciparum through the asexual IDC but a greater frequency of sexual gametocyte forms for $P$. vivax. In vitro survival, however, was markedly different, with a $77.3 \pm 6.5 \%$ survival rate observed for P. falciparum infected-normocytes at 44-h of culture compared to a $22.4 \pm 0.03$ survival rate for $P$. vivax-infected reticulocytes (Figs. 3c and $4 a$ ).

We subsequently assessed the osmotic stability of uninfected and $P$. falciparum trophozoite and schizonts stage-infected normocytes in 24- and 44-h cultures (the points at which $P$. vivax-infected reticulocytes were most destabilized). This analysis revealed no difference in the osmotic stability of $P$. falciparum infected (majority trophozoite) and uninfected-normocytes in 24h cultures and a reduction in P. falciparum infected-normocyte (majority schizonts) stability of $23.6 \pm 6.9 \%$ compared to uninfected normocytes in 44 -h cultures (Fig. 4b). This is in contrast to $P$. vivax, which had decreased reticulocyte stability by $74.0 \pm$ $14.6 \%$ by the time parasites had matured to the trophozoite form in 24-h cultures. Furthermore, direct comparison of the osmotic stability of $P$. falciparum-infected normocytes and $P$. vivaxinfected reticulocytes revealed that $P$. vivax-infected reticulocytes (max Lysis $_{50} 184.1 \pm 8.3,24$-h culture) are significantly less stable, $p<0.0003$, than P. falciparum-infected normocytes (max Lysis ${ }_{50}$ $107.8 \pm 4.7,44$-h culture) (Fig. $4 c$ ).

New permeability pathways increase $P$. vivax-infected reticulocyte permeability. New permeability pathways (NPPs) in related malaria parasites, $P$. falciparum and $P$. knowlesi, increase the permeability of the infected RBC to certain solutes ${ }^{6,7}$. To determine whether $P$. vivax possesses NPPs that are contributing to the decreased stability of the host reticulocyte, we assessed the sensitivity of Percoll-enriched cryopreserved Brazilian clinical $P$. vivax samples to the NPP antagonists D-sorbitol and L-alanine. For $P$. vivax-infected reticulocytes, we found that early stage parasites present in 8-h cultures were resistant to $\mathrm{D}$-sorbitol and $\mathrm{L}$ alanine lysis, while the appearance of stage III late trophozoite parasites in 16-h cultures corresponded with $P$. vivax-infected reticulocytes lysing in isotonic D-sorbitol (16-h-47.9 $\pm 9.0 \%$, $24-\mathrm{h}-45.2 \pm 9.2 \%$, and $44-\mathrm{h}-57.5 \pm 7.7 \%)$ and L-alanine solutions $(16-h-60.7 \pm 10.5 \%, 24-h-61.0 \pm 9.2 \%$, and $44-h-55.8 \pm$ $2.3 \%$ ). Finally, the NPP inhibitor, furosemide ${ }^{41}$, protected $P$. vivax-infected reticulocytes from $\mathrm{D}$-sorbitol and L-alanine lysis (Fig. 5a and b). The incomplete lysis of $P$. vivax-infected cells by D-sorbitol and L-alanine along with variation in the sensitivity of different $P$. vivax clinical isolates to $\mathrm{D}$-sorbitol and L-alanine lysis are potentially driven by (i) the high frequency of more stable gametocytes $(42.4 \pm 9.5 \%$ of $P$. vivax-infected reticulocytes in 
a.
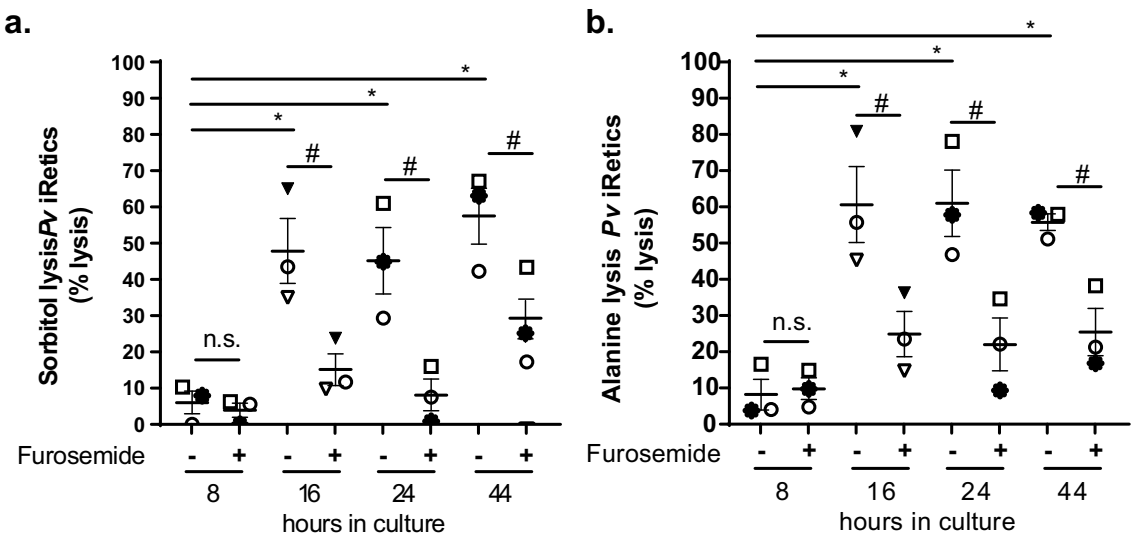

c.
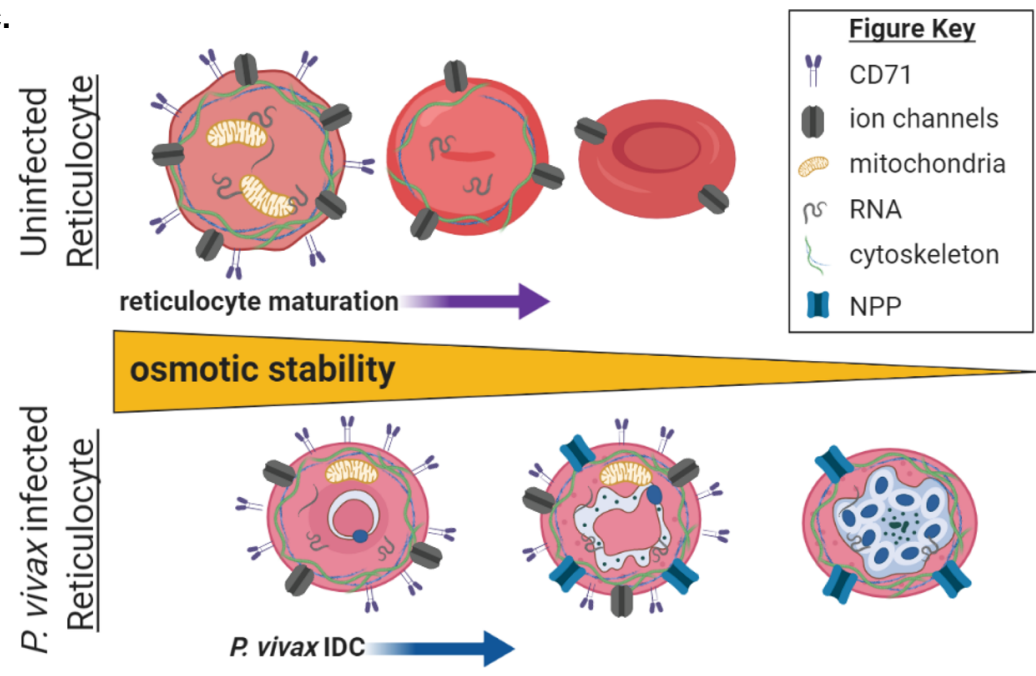

Fig. 5 P. vivax new permeability pathways increase the permeability of $\boldsymbol{P}$. vivax-infected reticulocytes. Sensitivity of cryopreserved Brazilian $P$. vivaxinfected reticulocytes to $\mathbf{a}$ D-sorbitol and $\mathbf{b}$ L-alanine lysis in the presence and absence of NPP inhibitor furosemide in 8-, 16-, 24-, and 44-h cultures ( $n=3$ ). Unique symbols indicate biological replicates. Horizontal lines and error bars represent mean \pm SEM. Hashtag and n.s., significant and no significant difference between furosemide treated and untreated $P$. vivax-infected reticulocyte lysis respectively (sorbitol: 8-h $p=0.6,16-\mathrm{h} p=0.02,24-\mathrm{h} p=0.04$, 44-h $p=0.02$ and alanine: 8-h $p=0.5,16-\mathrm{h} p=0.01,24-\mathrm{h} p=0.03,44-\mathrm{h} p=0.04$ ) using paired two-tailed Student's $t$-test. Asterisks, significant difference between $P$. vivax-infected reticulocyte lysis at 8-h and 16-, 24- and 44-h of culture (sorbitol: 16-h $p=0.01,24-\mathrm{h} p=0.02,44-\mathrm{h} p=0.004$ and alanine: $16-\mathrm{h} p=0.003,24-\mathrm{h} p=0.003,44-\mathrm{h} p=0.005)$ using unpaired two-tailed Dunnett's multiple comparisons test. c Model of the osmotic stability dynamics within the reticulocyte compartment and the impact of $P$. vivax infection on reticulocyte osmotic stability. Created with BioRender.com.

44-h cultures) ${ }^{42,43}$ (Fig. 3c), (ii) variable NPP activity in different $P$. vivax isolates, or (iii) $P$. vivax being less sensitive to D-sorbitol lysis than $P$. falciparum ${ }^{44,45}$.

\section{Discussion}

Structural stability of $P$. vivax-infected reticulocytes, critical for the successful propagation of blood-stage malaria infections, has not been thoroughly assessed due to lack of appropriate methodology compatible with $P$. vivax's unique and challenging biology. To study the impact of $P$. vivax infection on the structural integrity of the host reticulocyte, we adapted the osmotic stability assay to be analyzed by flow cytometry. This permitted us to work with the limited cell numbers and heterogeneity characteristic of both the reticulocyte compartment to which $P$. vivax infection is restricted as well as $P$. vivax-infected clinical samples. In the proceeding studies, we observed that the youngest $\mathrm{CD} 71+$ reticulocytes, which $P$. vivax preferentially invades, were the most osmotically stable of enucleated RBC subsets and that $P$. vivax-infected reticulocytes were less stable than uninfected $\mathrm{CD} 71+$ reticulocytes. These results indicate that $P$. vivax severely compromises the structural integrity of the host reticulocyte. In addition to its use in determining the osmotic stability of erythroid precursor and malaria infected-RBC samples, the single-cell resolution of the flow cytometry osmotic stability assay provides a means for tracking the impact of erythropoiesis perturbations on overall RBC osmotic stability and examining osmotic stability dynamics within heterozygous hematological diseases such as sickle cell and G6PD deficiency.

The decreasing osmotic stability we observed in the later stages of erythropoiesis and then continuing through reticulocyte maturation is likely driven by three major changes occurring to erythroid cells during their differentiation: (i) intracellular solutes changes due to changing membrane transporter abundance and activity 46,47 , (ii) cytoskeleton remodeling ${ }^{48,49}$, and (iii) the progressive reduction in the surface to volume ratio driven by membrane loss 50 . The observation by us and others ${ }^{18-20}$ that reticulocytes are more resistant to osmotic lysis than normocytes is seemingly incongruent with previous reports of membrane instability in reticulocytes ${ }^{51}$. This discrepancy is likely due to either (i) the factors that determine osmotic stability, like higher surface area to volume ratio and greater abundance and activity of membrane transporters in reticulocytes, are more dominant than reticulocyte membrane instability, or (ii) the biology underlying membrane instability and osmotic stability are independent of one another. Ultimately, the factors that determine the stability of erythroid precursors and 
reticulocytes are likely multifactorial and highly dynamic. Finally, we and others ${ }^{19}$ have found that in vitro differentiated reticulocytes are less stable than ex vivo reticulocytes. Interestingly the addition of cholesterol improved the stability of in vitro cultured reticulocytes ${ }^{19}$, suggesting that approaches pursuing the determinants of erythroid progenitor and reticulocyte osmotic stability may yield strategies for generating more stable and consequently more viable RBCs from in vitro cultures.

Our study revealed that $P$. vivax infection decreased the osmotic stability of the host reticulocyte. Though consistent with what has been observed for other Plasmodium spp. $6,7,9,52,53, P$. vivax notably decreased reticulocyte osmotic stability to a significantly greater degree than the more extensively studied $P$. falciparum decreased normocyte osmotic stability. Variation in the instability induced by Plasmodium spp. parasites has also been observed for P. knowlesiinfected normocytes, that are similarly less stable than P. falciparum-infected normocytes ${ }^{44}$. Comparing this data set with ours however, we estimate $P$. vivax-infected reticulocytes are the least stable of the three Plasmodium spp., being $70 \%$ less stable than $P$. falciparum-infected normocytes whereas $P$. knowlesi-infected normocytes are 30\% less stable than P. falciparum-infected normocytes. Moreover, $P$. vivax-infected reticulocyte instability is on par with RBC instability observed for hemolytic anemias like HS. Together these findings identify a new parameter of malaria blood stage pathophysiology that differs between Plasmodium spp. that has the potential to reveal avenues to species-specific therapeutics.

The observation that $P$. vivax compromises the osmotic stability of the host reticulocyte is consistent with the observation that the majority of late stage $P$. vivax-infected reticulocytes lysed when passed through $2 \mu \mathrm{m}$ microfluidic channels ${ }^{16}$, and suggest that the instability induced by $P$. vivax significantly increases the risk of premature hemolysis of infected reticulocytes. The destabilization of reticulocytes by $P$. vivax infection is also consistent with the precipitous loss of $P$. vivax-infected reticulocytes from in vitro culture observed in this as well as previous studies ${ }^{33-36}$. That said, as $P$. vivax is clearly capable of propagating its blood stage infection in vivo, $P$. vivax likely has strategies for coping with the compromised structural integrity of the host reticulocyte. Future studies will provide important insight into (i) the in vivo consequences of the compromised structural integrity of infected-reticulocytes and how $P$. vivax is able to circumvent the reduced fitness of its host cell to propagate successful blood stage infections and (ii) strategies for stabilizing $P$. vivax-infected reticulocytes in vitro to help facilitate culture adaptation of $P$. vivax.

Intriguingly, clinical observations that $P$. vivax infection results in 4 times higher clearance of uninfected RBCs than P. falciparum ${ }^{54,55}$ suggests that $P$. vivax infection not only more severely compromises the host reticulocyte it infects but surrounding RBCs as well. As a consequence of the global destabilization of host RBCs, the frequency and severity of $P$. vivax associated anemia is on par with $P$. falciparum $^{55-57}$ associated anemia despite the reticulocyte tropism of $P$. vivax resulting in a lower parasite biomass than $P$. falciparum infections ${ }^{58}$. This anemia is likely the consequence of intravascular hemolysis, opsonophagocytosis driven extravascular hemolysis and dyserythropoiesis ${ }^{59,60}$. The degree to which each of these contributes to $P$. vivax associated anemia remain to be determined.

In our study we found that $P$. vivax NPPs are associated with reduced stability of host reticulocytes. This raises several questions including: (i) are P. vivax NPPs required for the acquisition of essential nutrients from the host serum and (ii) what host and or parasite transporters are responsible for the observed NPP activity. The presence of NPP activity in P. vivax-infected reticulocytes suggests some overlap between the underlying factors responsible for the reduced osmotic stability of $P$. falciparum and $P$. vivax-infected cells. In P. falciparum, Clag3.1 and Clag3.2 have been identified as parasite mediators of NPP activity in infected
$\mathrm{RBCs}^{61,62}$, and $P$. vivax possesses a clag family of genes ${ }^{63}$. This raises the possibility of therapeutically targeting $P$. vivax-infected reticulocytes by inhibiting Clag function, as has been done for $P$. falciparum $^{64,65}$

NPPs are unlikely to be the single variable contributing to the reduced stability of $P$. vivax-infected reticulocytes. Previously reported shedding of microvesicles from $P$. vivax-infected reticulcoytes $^{66}$ and $100 \mathrm{~nm}$ "holes" in $P$. vivax-infected reticulocytes ${ }^{13}$ could each reduce osmotic stability by decreasing surface to volume ratio and increasing permeability of $P$. vivaxinfected reticulocytes. Determining the biology underlying the compromised structural integrity of infected reticulocytes has the potential to yield targets against which therapeutics could be developed. Finally, considering the compromised stability of $P$. vivax-infected reticulocytes in the context of the protection from Plasmodium infection afforded by RBC polymorphisms such as HS, thalassemia, and G6PD deficiency, it is possible that a mechanism underlying protection from $P$. vivax may be premature hemolysis of infected RBCs.

Together our data support a model in which $P$. vivax referred CD71+ reticulocytes are more osmotically stable than CD71reticulocytes and normocytes, but, upon $P$. vivax infection and subsequent maturation, the infected reticulocyte undergoes a precipitous loss of osmotic stability. Moreover, the magnitude of instability induced by $P$. vivax is close to the instability observed for hemolytic anemias like HS. Additionally, the onset of NPP activity corresponds with the decreasing osmotic stability of infected reticulocytes (Fig. 5c). As a result, $P$. vivax-infected reticulocytes likely exhibit increased rates of intravascular hemolysis and premature clearance from circulation similar to what is observed for RBCs from individuals with hemolytic anemias. Future studies will focus on the mechanisms underlying these changes in osmotic stability that impact the structural integrity of the host reticulocyte and the consequences of this instability on in vivo survival. Finally, identifying strategies for stabilizing $P$. vivax-infected reticulocyte could prove key to culture-adapting $P$. vivax, while strategies to further destabilize $P$. vivax-infected reticulocytes could conversely yield novel blood stage therapies for $P$. vivax.

\section{Methods}

Ethics approval. For HX, bone marrow aspirate, and Brazilian and Indian clinical $P$. vivax samples, we confirm that all relevant ethical regulations were complied with. For HX and human bone marrow samples negative for blasts or diserythropoietic conditions, informed consent was obtained for all patients and samples were collected under the approval of the Boston Children's Hospital Institutional Review Board (IRB04-02-017R) and were de-identified for use in this study. We obtained human IRB waivers for the use of the de-identified parasite samples from the Harvard School of Public Health Office of Human Research Administration for Brazil (IRB21410-101) and for India (IRB17-1071). For Brazilian clinical $P$. vivax samples, informed consent was obtained from all patients. Study protocols for Brazilian parasite sample collection were approved by the IRB of the Institute of Biomedical Sciences, University of São Paulo, Brazil (IRB 1169/ CEPSH, 2014). For Indian P. vivax samples, informed consent was obtained from all patients. Study protocols for Indian parasite sample collection were approved by the ethics boards at Goa Medical College and Hospital (GMC) (no number assigned), University of Washington (42271), the Division of Microbiology and Infectious Diseases of the US National Institutes of Health (NIH) (11-0074) and the Government of India Health Ministry Screening Committee (HMSC) for collection of the Indian isolates.

Parasite sample collection. Brazilian clinical $P$. vivax samples were collected in the town of Mâncio Lima, Acre State, leukodepleted with BioR 01 Plus filters, cryopreserved with glycerolyte, and stored in liquid nitrogen. The collections were performed in the context of a randomized, open-label clinical trial (NCT02691910). Indian P. vivax samples were collected at Goa Medial College and Hospital in Bambolim, Goa, in conjunction with the Malaria Evolution in South Asia International Center of Excellence in Malaria Research and the University of Washington and leukodpleted with CF11.

Bone marrow and parasite enrichment. Bone marrow aspirates, cryopreserved Brazilian clinical $P$. vivax samples and $P$. falciparum $3 D 7 P 2 G 12$ were enriched on 
$1.080 \mathrm{~g} / \mathrm{mL} \mathrm{KCl} \mathrm{high} \mathrm{Percoll} \mathrm{gradients}{ }^{33}$. Briefly, $2 \mathrm{~mL}$ of re-suspended cells (up to $25 \%$ hematocrit) were layered on $3 \mathrm{~mL} 1.080 \mathrm{~g} / \mathrm{mL} \mathrm{KCl}$ high Percoll gradient and spun for $15 \mathrm{~min}$ at $1200 \times \mathrm{g}$. Subsequently, the interface was removed, washed, and applied to assays. For $P$. falciparum 3D7 P2G12 cultures, the interface and pellet were washed and recombined before being applied to assays.

CD34+ in vitro RBC differentiation. CD34+ hematopoetic stem cells (HSCs) purchased commercially (Lonza) were differentiated in vitro following the established three-step differentiation protocol ${ }^{19,32}$ in Iscove's liquid medium (Biochrom) with $5 \%$ human plasma (Octapharma). Giemsa-stained cytospins were prepared and a minimum 200 cells per slide $(\times 1000$ magnification $)$ were called as either proerythroblast, basophilic normoblast, polychromatic normoblast, orthochromatic normoblast, or reticulocyte. Images were taken at $\times 1000$ magnification using an Excelis HD Camera attached to an Olympus BX40 microscope.

P. vivax and $\boldsymbol{P}$. falciparum in vitro culture. Indian and Brazilian clinical $P$. vivax samples and P. falciparum 3D7 P2G12 were cultured at $100 \times 10^{6}$ cells per $\mathrm{mL}$ in IMDM (Gibco) containing $10 \% \mathrm{AB}^{+}$heat-inactivated sera and $50 \mu \mathrm{g} / \mathrm{mL}$ gentamicin at $37^{\circ} \mathrm{C}$ in $5 \% \mathrm{CO}_{2}, 1 \% \mathrm{O}_{2}$, and $94 \% \mathrm{~N}_{2}{ }^{33}$. Hemacolor-stained cytospins were prepared and 200 cells per slide ( $\times 1000$ magnification) were called as either asexual stage (I-V) or gametocyte ${ }^{67}$. Images of cytospins were taken at $\times 1000$ magnification using an Excelis HD Camera attached to an Olympus BX40 microscope. Osmotic stability assays for non-cryopreserved clinical Indian P. vivax samples were performed locally at Goa Medical College where samples were collected while cryopreserved clinical Brazilian P. vivax samples were shipped from São Paulo Brazil to Boston, MA where they were thawed and osmotic stability assays were performed.

Flow cytometry. For the described flow cytometry experiments, $1 \times 10^{7}$ cells were washed with flow buffer prior to staining with combinations of the following fluorophores: Thiazole Orange, 1:1000 (Thermo Fisher Scientific), Vybrant DyeCycle Violet, 1:5000 (Thermo Fisher Scientific), Vybrant DyeCycle Green, 1:5000 (Thermo Fisher Scientific), propidium iodide, 1:500 (Thermo Fisher Scientific), $a-$ CD71-APC, 1:25 (Miltenyi Biotech), a-GypA-FITC, 1:100 (Stem Cell Technologies), and FITC-Phalloidin, 1:200 (Thermo Fisher Scientific). Bone marrow and in vitro cultured RBC samples were stained at $4^{\circ} \mathrm{C}$ for $20 \mathrm{~min}$ and $P$. vivax and $P$. falciparum samples at $37^{\circ} \mathrm{C}$ for $20 \mathrm{~min}$. Prior to analysis by flow cytometry, AccuCheck counting beads (Thermo Fisher Scientific) were added to each sample. All flow cytometry experiments were acquired on a Miltenyi MACSQuant instrument equipped with MACSQuantify version 2.11 and 405, 488, and 638-nm lasers and a minimum of 200 events were collected for each cell population being analyzed for the no lysis control sample. All subsequent gating and analysis was based on the no-lysis control sample. As lysis in subsequent samples resulted in ultimate destruction of the cell populations, this minimum event count was not able to be achieved for all samples. For all conditions analyzed, a minimum of 2000 events were collected for cell counting beads. Samples with parasitemia and or reticulocytemia below $0.05 \%$ were considered below the limit of detection. Data were analyzed using FlowJo (Version 10.4).

Osmotic stability assays. Phosphate buffer saline lysis solutions ${ }^{22}$ ranging from 300 to 0 mOsm (distilled water) were made by serial dilution and final osmolarity measured by vapor pressure osmometer (Wescor Vapro 5520). For flow cytometric osmotic stability assays, $1 \times 10^{6}$ cells (stained with appropriate fluorescent dyes and or antibodies) were incubated at $5 \times 10^{6}$ cells $/ \mathrm{mL}$ in lysis solutions ranging from $\sim 300$ to $0 \mathrm{mOsm}$ (distilled water) for $10 \mathrm{~min}$ at $37^{\circ} \mathrm{C}$. Lysis was stopped with $4 \times$ volume of quenching solution (1:10 AccuCheck Counting Beads (Thermo Fisher Scientific) to flow buffer). Only cells with normal flow cytometric FSC/SSC profiles (as defined by the control $300 \mathrm{mOsm}$ condition) were considered to be intact. For assays containing nucleated cells, nuclei and dead cells were additionally excluded from the intact cell population. The ratio of beads to intact cells was used to calculate the absolute number of cells remaining in each lytic condition and percent lysis was determined by normalization to the $300 \mathrm{mOsm}$ control condition $(0 \%$ lysis). For hemoglobin absorbance osmotic stability assays, cells were lysed as described above before $4 \times$ volume of flow buffer was added to stop lysis. Cells were pelleted and the absorbance $(380,415,450$, and $540 \mathrm{~nm})$ of supernatants measured on a Spectramax M5 plate reader. The amount of hemoglobin present in supernatants was calculated by the Harboe method ${ }^{68}$. Percent lysis was determined by normalization to the $300 \mathrm{mOsm}$ control condition ( $0 \%$ lysis).

RBC ghost assay. For RBC ghost detection by flow cytometry, FITC conjugated phalloidin was added to all lysis and quenching solutions. For RBC ghost detection by immunofluorescence microscopy, RBCs suspended in PBS containing FITCPhalloidin were allowed to settle on a coverslip before a hypotonic solution (57 mOsm) containing FITC-Phalloidin was added and RBC lysis and ghost formation examined by live video microscopy on a Zeiss AxioObserver.Z1. Images were taken at $\times 630$ magnification on a Zeiss AxioObserver.Z1 inverted fluorescent microscope coupled to an AxioCam MRm camera. Images were processed using Fiji ImageJ $1.52 \mathrm{p}$ software.
Sorbitol and alanine hemolysis assay. $P$. vivax cultures were incubated at $5 \times 10^{6}$ cells $/ \mathrm{mL}$ for $30 \mathrm{~min}$ at $37^{\circ} \mathrm{C}$ in flow buffer, $280 \mathrm{mM}$ sorbitol with $1 \%$ BSA, or 280 $\mathrm{mM}$ alanine with $1 \% \mathrm{BSA}$ in the presence or absence of $100 \mu \mathrm{M}$ furosemide. Following incubation, cells were washed twice with flow buffer, and stained for flow cytometry analysis.

Statistics and reproducibility. Lysis $_{50}$ values derived from least-squares regression fit curves of normalized data were used to assess the reproducibility of results from biological replicates. Biological replicates shown for (i) normal RBCs, (ii) HX RBCs, (iii) bone marrow aspirates, (iv) CD34+ erythroid culture, and (v) Brazilian and Indian $P$. vivax samples are biologically independent samples, and experiments were performed in batches with 1-3 samples being assessed in a given experiment For P. falciparum 3D7 (P2G12 clone) biological replicates are five independently cryopreserved $P$. falciparum 3D7 (P2G12 clone) samples thawed sequentially so that experiments were performed independently. Prism (GraphPad Prism8) and Stata (version 16.0, StataCorp LLC., College Station, TX, USA) were used to test for statistical significance.

Reporting summary. Further information on research design is available in the Nature Research Reporting Summary linked to this article.

\section{Data availability}

All data generated or analyzed during this study are included in this published article (and its Supplementary information files). A reporting summary for this article is available as a Supplementary information file. The P. falciparum 3D7-P2G12 clone used in this study will be made available upon request. All additional samples were clinical ex vivo samples for which we do not have additional material to supply. Source data are provided with this paper.

Received: 4 August 2020; Accepted: 17 February 2021; Published online: 12 March 2021

\section{References}

1. Uzoigwe, C. The human erythrocyte has developed the biconcave disc shape to optimise the flow properties of the blood in the large vessels. Med. Hypotheses 67, 1159-1163 (2006).

2. Zarychanski, R. et al. Mutations in the mechanotransduction protein PIEZO1 are associated with hereditary xerocytosis. Blood 120, 1908-1915 (2012).

3. Eber, S. W. et al. Ankyrin-1 mutations are a major cause of dominant and recessive hereditary spherocytosis. Nat. Genet. 13, 214-218 (1996).

4. King, M.-J. \& Zanella, A. Hereditary red cell membrane disorders and laboratory diagnostic testing. Int. J. Lab. Hematol. 35, 237-243 (2013).

5. Glenister, F. K., Coppel, R. L., Cowman, A. F., Mohandas, N. \& Cooke, B. M. Contribution of parasite proteins to altered mechanical properties of malariainfected red blood cells. Blood 99, 1060-1063 (2002).

6. Ginsburg, H., Krugliak, M., Eidelman, O. \& Ioav Cabantchik, Z. New permeability pathways induced in membranes of Plasmodium falciparum infected erythrocytes. Mol. Biochem. Parasitol. 8, 177-190 (1983).

7. Lisk, G. \& Desai, S. A. The plasmodial surface anion channel is functionally conserved in divergent malaria parasites. Eukaryot. Cell 4, 2153-2159 (2005).

8. Safeukui, I. et al. Retention of Plasmodium falciparum ring-infected erythrocytes in the slow, open microcirculation of the human spleen. Blood 112, 2520-2528 (2008).

9. Lew, V. L., Macdonald, L., Ginsburg, H., Krugliak, M. \& Tiffert, T. Excess haemoglobin digestion by malaria parasites: a strategy to prevent premature host cell lysis. Blood Cells Mol. Dis. 32, 353-359 (2004).

10. Buffet, P. A. et al. The pathogenesis of Plasmodium falciparum malaria in humans: insights from splenic physiology. Blood 117, 381-392 (2011).

11. Howes, R. E. et al. Global epidemiology of Plasmodium vivax. Am. J. Trop. Med. Hyg. 95, 15-34 (2016).

12. Craik, R. A note on the erythrocytes in malaria. Lancet 195, 1110 (1920).

13. Malleret, B. et al. Plasmodium vivax: restricted tropism and rapid remodeling of CD71-positive reticulocytes. Blood 125, 1314-1324 (2015).

14. Gruszczyk, J. et al. Transferrin receptor 1 is a reticulocyte-specific receptor for Plasmodium vivax. Science 359, 48-55 (2018)

15. Suwanarusk, R. et al. The deformability of red blood cells parasitized by Plasmodium falciparum and P. vivax. J. Infect. Dis. 189, 190-194 (2004).

16. Handayani, S. et al. High deformability of Plasmodium vivax-infected red blood cells under microfluidic conditions. J. Infect. Dis. 199, 445-450 (2009).

17. Cranston, H. A. et al. Plasmodium falciparum maturation abolishes physiologic red cell deformability. Science 223, 400-403 (1984).

18. Grimberg, B. T. et al. Increased reticulocyte count from cord blood samples using hypotonic lysis. Exp. Parasitol. 132, 304-307 (2012). 
19. Bernecker, C. et al. Cholesterol deficiency causes impaired osmotic stability of cultured red blood cells. Front. Physiol. 10, 1529 (2019).

20. Tada, K., Watanabe, Y. \& Miyazawa, H. [Studies on the reticulocyte. I. The $\mathrm{MCV}, \mathrm{MCH}, \mathrm{MCHC}$ and osmotic fragility of the reticulocyte]. Paediatr. Jpn 66, 263-265 (1962).

21. Niskanen, E. \& Cline, M. J. Differentiation of subpopulations of human and murine hemopoietic stem cells by hypotonic lysis. J. Clin. Investig. 65, 285-289 (1980).

22. Beutler, E., Kuhl, W. \& West, C. The osmotic fragility of erythrocytes after prolonged liquid storage and after reinfusion. Blood 59, 1141-1147 (1982).

23. Rudzinska, M. A., Trager, W. \& Bray, R. S. Pinocytotic uptake and the digestion of hemoglobin in malaria parasites. J. Protozool. 12, 563-576 (1965).

24. Smith, D. W. Reticulocyte transfer RNA and hemoglobin synthesis. Science 190, 529-535 (1975)

25. Ciepiela, O., Adamowicz-Salach, A., Zgodzińska, A., Łazowska, M. \& Kotuła, I. Flow cytometric osmotic fragility test: Increased assay sensitivity for clinical application in pediatric hematology. Cytometry Part B 94, 189-195 (2017).

26. Yamamoto, A. et al. Flow cytometric analysis of red blood cell osmotic fragility. J. Lab. Autom. 19, 483-487 (2014).

27. Nobre, C. S., Silva, J. A., Jácomo, R. H., Nery, L. F. A. \& Barra, G. B. Flow cytometric analysis of erythrocytes osmotic fragility in hereditary spherocytosis: a case-controlled study evaluating the best anticoagulant, sample pre-treatment and $\mathrm{NaCl}$ concentration for reliable screening of this red blood cell membrane disorder. Cytometry Part B 94, 910-917 (2018).

28. Warang, P., Gupta, M., Kedar, P., Ghosh, K. \& Colah, R. Flow cytometric osmotic fragility-an effective screening approach for red cell membranopathies. Cytometry Part B80, 186-190 (2011).

29. Bermúdez, M. et al. Receptor-ligand and parasite protein-protein interactions in Plasmodium vivax: analysing rhoptry neck proteins 2 and 4. Cell. Microbiol. 20, e12835 (2018).

30. Fischbach, F. T. A Manual of Laboratory and Diagnostic Tests (Wolters Kluwer Health, 2015).

31. Godal, H. C., Elde, A. T., Nyborg, N. \& Brosstad, F. The normal range of osmotic fragility of red blood cells. Scand. J. Haematol. 25, 107-112 (1980).

32. Giarratana, M.-C. et al. Ex vivo generation of fully mature human red blood cells from hematopoietic stem cells. Nat. Biotechnol. 23, 69-74 (2005).

33. Rangel, G. W. et al. Enhanced ex vivo Plasmodium vivax intraerythrocytic enrichment and maturation for rapid and sensitive parasite growth assays. Antimicrob. Agents Chemother. 62, e02519-17 (2018).

34. Rangel, G. W. et al. Plasmodium vivax transcriptional profiling of low input cryopreserved isolates through the intraerythrocytic development cycle. PLoS Negl. Trop. Dis. 14, e0008104 (2020).

35. Borlon, C. et al. Cryopreserved Plasmodium vivax and cord blood reticulocytes can be used for invasion and short term culture. Int. J. Parasitol. 42, 155-160 (2012).

36. Tetsuya, F., Juliana, M. S., Chetan, E. C., Thomas, E. W. \& Timothy, T. S. Reticulocytes from cryopreserved erythroblasts support Plasmodium vivax infection in vitro. Parasitol. Int. 63, 278-284 (2013).

37. Archer, N. M. et al. Hereditary xerocytosis revisited. Am. J. Hematol. 89, 1142-1146 (2014).

38. Khalid, U. Osmotic fragility of erythrocytes: reference range, interpretation, collection and panels. Medscape. https://emedicine.medscape.com/article/2085814 (2020).

39. Gronowicz, G., Swift, H. \& Steck, T. L. Maturation of the reticulocyte in vitro. J. Cell. Sci. 71, 177-197 (1984).

40. Buchholz, K. et al. A high-throughput screen targeting malaria transmission stages opens new avenues for drug development. J. Infect. Dis. 203, 1445-1453 (2011).

41. Kirk, K., Horner, H. A., Elford, B. C., Ellory, J. C. \& Newbold, C. I. Transport of diverse substrates into malaria-infected erythrocytes via a pathway showing functional characteristics of a chloride channel. J. Biol. Chem. 269, 3339-3347 (1994).

42. Saul, A., Graves, P. \& Edser, L. Refractoriness of erythrocytes infected with Plasmodium falciparum gametocytes to lysis by sorbitol. Int. J. Parasitol. 20, 1095-1097 (1990).

43. Shaw-Saliba, K. et al. Infection of laboratory colonies of Anopheles mosquitoes with Plasmodium vivax from cryopreserved clinical isolates. Int. J. Parasitol. 46, 679-683 (2016)

44. Liu, B. et al. Multimodal analysis of Plasmodium knowlesi-infected erythrocytes reveals large invaginations, swelling of the host cell, and rheological defects. Cell. Microbiol. 21, e13005 (2019).

45. van Schalkwyk, D. A., Moon, R. W., Blasco, B. \& Sutherland, C. J. Comparison of the susceptibility of Plasmodium knowlesi and Plasmodium falciparum to antimalarial agents. J. Antimicrob. Chemother. 72, 3051-3058 (2017).

46. Blanc, L. et al. The water channel aquaporin-1 partitions into exosomes during reticulocyte maturation: implication for the regulation of cell volume. Blood 114, 3928-3934 (2009).

47. Pan, D. et al. $\mathrm{K}-\mathrm{Cl}$ cotransporter gene expression during human and murine erythroid differentiation. J. Biol. Chem. 286, 30492-30503 (2011).
48. Griffiths, R. E. et al. The ins and outs of human reticulocyte maturation: autophagy and the endosome/exosome pathway. Autophagy 8, 1150-1151 (2012).

49. Koury, S. T., Koury, M. J. \& Bondurant, M. C. Cytoskeletal distribution and function during the maturation and enucleation of mammalian erythroblasts. J. Cell Biol. 109, 3005-3013 (1989).

50. Mohandas, N. \& Groner, W. Cell membrane and volume changes during red cell development and aging. Ann. N. Y. Acad. Sci. 554, 217-224 (1989).

51. Waugh, R. E. Reticulocyte rigidity and passage through endothelial-like pores. Blood 78, 3037-3042 (1991).

52. Fogel, B. J., Shields, C. E. \& Von Doenhoff, A. E. The osmotic fragility of erythrocytes in experimental malaria. Am. J. Trop. Med. Hyg. 15, 269-275 (1966).

53. Seed, T. M., Brindley, D., Aikawa, M. \& Rabbege, J. Plasmodium berghei: osmotic fragility of malaria parasites and mouse host erythrocytes. Exp. Parasitol. 40, 380-390 (1976)

54. Price, R. N. et al. Factors contributing to anemia after uncomplicated falciparum malaria. Am. J. Trop. Med. Hyg. 65, 614-622 (2001).

55. Collins, W. E., Jeffery, G. M. \& Roberts, J. M. A retrospective examination of anemia during infection of humans with Plasmodium vivax. Am. J. Trop. Med. Hyg. 68, 410-412 (2003).

56. Douglas, N. M. et al. Major burden of severe anemia from non-falciparum malaria species in Southern Papua: a hospital-based surveillance study. PLoS Med. 10, e1001575 (2013). discussion e1001575.

57. Douglas, N. M. et al. The anaemia of Plasmodium vivax malaria. Malar. J. 11, 135 (2012).

58. Barber, B. E. et al. Parasite biomass-related inflammation, endothelial activation, microvascular dysfunction and disease severity in vivax malaria. PLoS Pathog. 11, e1004558 (2015).

59. Anstey, N. M., Russell, B., Yeo, T. W. \& Price, R. N. The pathophysiology of vivax malaria. Trends Parasitol. 25, 220-227 (2009).

60. Anstey, N. M., Douglas, N. M., Poespoprodjo, J. R. \& Price, R. N. Plasmodium vivax: clinical spectrum, risk factors and pathogenesis. Adv. Parasitol. 80, 151-201 (2012).

61. Nguitragool, W. et al. Malaria parasite clag3 genes determine channelmediated nutrient uptake by infected red blood cells. Cell 145, 665-677 (2011).

62. Mira-Martínez, S. et al. Epigenetic switches in clag3 genes mediate blasticidin S resistance in malaria parasites. Cell. Microbiol. 15, 1913-1923 (2013).

63. Dharia, N. V. et al. Whole-genome sequencing and microarray analysis of ex vivo Plasmodium vivax reveal selective pressure on putative drug resistance genes. Proc. Natl Acad. Sci. USA 107, 20045-20050 (2010).

64. Dickerman, B. K. et al. Identification of inhibitors that dually target the new permeability pathway and dihydroorotate dehydrogenase in the blood stage of Plasmodium falciparum. Sci. Rep. 6, 37502 (2016).

65. Pain, M. et al. Synergistic malaria parasite killing by two types of plasmodial surface anion channel inhibitors. PLoS ONE 11, e0149214 (2016).

66. Toda, H. et al. Plasma-derived extracellular vesicles from Plasmodium vivax patients signal spleen fibroblasts via NF-kB facilitating parasite cytoadherence. Nat. Commun. 11, 2761 (2020)

67. Lim, C. et al. Reticulocyte preference and stage development of Plasmodium vivax isolates. J. Infect. Dis. 214, 1081-1084 (2016).

68. Harboe, M. A method for determination of hemoglobin in plasma by near-ultraviolet spectrophotometry. Scand. J. Clin. Lab. Investig. 11, 66-70 (1959).

\section{Acknowledgements}

We thank Juliet Imperial, Flow Cytometry Laboratory, Boston Children's Hospital for providing bone marrow samples and Steven Staffa, MS for providing statistical analysis consultation. This study was supported by U.S. NIH/NHLBI F32 HL136173, NIH R01 AI140751, NIH R01 HL139337, and NIH/NIAID MESA-ICEMR Program Project U19 AI89688, the Canadian Institutes of Health Research Postdoctoral Fellowship, and a Conselho Nacional de Desenvolvimento Científico e Tecnológico (CNPq) research scholarship. The clinical trial that originated samples from Brazil was supported by the Ministry of Health of Brazil and the CNPq, grant 404067/2012-3. The funders had no role in study design, data collection and interpretation, or the decision to submit the work for publication.

\section{Author contributions}

M.A.C., U.K., G.W.R., M.T.D.-Experimental design, execution, and data analysis. L.C., A.M., E.G., P.K.R., C.B., M.U.F.-Clinical management of uninfected and infected blood sample collection, including patient management and ethical clearance. M.A.C., U.K., G.W.R., C.B., M.T.D.-Data interpretation and manuscript writing.

\section{Competing interests}

The authors declare no competing interests. 


\section{Additional information}

Supplementary information The online version contains supplementary material available at https://doi.org/10.1038/s41467-021-21886-x.

Correspondence and requests for materials should be addressed to M.T.D.

Peer review information Nature Communications thanks Carla Cerami, Steven Kho, and Louis Miller for their contribution to the peer review of this work. Peer reviewer reports are available.

Reprints and permission information is available at http://www.nature.com/reprints

Publisher's note Springer Nature remains neutral with regard to jurisdictional claims in published maps and institutional affiliations. (c) (1) Open Access This article is licensed under a Creative Commons Attribution 4.0 International License, which permits use, sharing, adaptation, distribution and reproduction in any medium or format, as long as you give appropriate credit to the original author(s) and the source, provide a link to the Creative Commons license, and indicate if changes were made. The images or other third party material in this article are included in the article's Creative Commons license, unless indicated otherwise in a credit line to the material. If material is not included in the article's Creative Commons license and your intended use is not permitted by statutory regulation or exceeds the permitted use, you will need to obtain permission directly from the copyright holder. To view a copy of this license, visit http://creativecommons.org/ licenses/by/4.0/.

(C) The Author(s) 2021 